\title{
Effect of Mixed Layer Crystallinity on the Performance of Mixed Heterojunction Organic Photovoltaic Cells
}

\author{
Byeongseop Song, Cedric Rolin, Jeramy D. Zimmerman, and Stephen R. Forrest*
}

State-of-the-art small molecular weight organic solar cells rely on multilayer structures whose morphology must be engineered at the nanoscale. ${ }^{[1-5]}$ It follows, therefore, that growth methods enabling control over film morphology and layering schemes are required to achieve this objective. One such method is organic vapor phase deposition (OVPD), where organic molecules are transported by a hot inert carrier gas from the source to a cooled substrate where they condense to form a thin film. ${ }^{[6,7]}$ Compared to conventional vacuum thermal evaporation (VTE), the use of a carrier gas in OVPD dramatically changes many aspects of the film deposition kinetics. For example, the presence of carrier gas molecules in the vicinity of the substrate leads to a reduced molecular mean free path. ${ }^{[8-10]}$ Furthermore, the formation of complex multilayers and mixed or doped layers are enabled by the precise control over gas flow rates, source temperatures, background pressure, and substrate temperature. ${ }^{[11]}$ And finally, OVPD is capable of high speed film growth with efficient material utilization and thickness uniformity over large substrate areas. ${ }^{[12,13]}$ Indeed, OVPD is particularly useful in the growth of complex morphologies such as those inherent in mixed donor/acceptor heterojunctions, These strutures have been shown to maximize the efficiency of exciton dissociation by distributing the donor-acceptor interface throughout the photoactive OPV layers. ${ }^{[1-16]}$ The morphological control afforded by OVPD can be engineered to minimize photogenerated charge recombination often incurred in the mixed region. ${ }^{[8,17,18]}$ In this work, we demonstrate organic photovoltaic (OPV) cells based on a nanocrystalline mixed tetraphenyldibenzoperiflanthen (DBP): $\mathrm{C}_{70}$ heterojunction grown by OVPD with a power conversion efficiency, $P C E=6.7 \pm 0.2 \%$, compared to $6.2 \pm 0.2 \%$ for analogous, optimized devices grown by VTE. Due to the lower electrical resistance of the nanocrystalline layers

\author{
B. Song, Dr. C. Rolin, Dr. J. D. Zimmerman, \\ Prof. S. R. Forrest \\ Department of Electrical Engineering \\ and Computer Science \\ University of Michigan \\ Ann Arbor, MI 48109, USA \\ E-mail: stevefor@umich.edu \\ Dr. C. Rolin \\ Large Area Electronics Group \\ IMEC Kapeldreef \\ 75 B-3001, Leuven, Belgium \\ Prof. S. R. Forrest \\ Department of Physics \\ Materials Science and Engineering \\ University of Michigan \\ Ann Arbor, MI 48109, USA
}

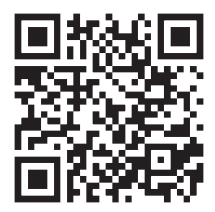

DOI: 10.1002/adma.201305099 formed via OVPD, the active region thickness can be almost double those grown by VTE. The increased cell thickness has the potential to ease manufacturing tolerances by reducing the occurrence of shorts due to pinholes often encountered in thinner cells. ${ }^{[19-21]}$ In addition, the material utilization efficiency of OVPD is generally significantly higher than VTE, ${ }^{[22,23]}$ thereby the increased thickness of the organic layer should not negatively impact the total cost of device fabrication.

Mixed heterojunction OPVs were fabricated with the following structure: Glass/indium tin oxide (ITO) anode $/ \mathrm{MoO}_{3}$ $(10 \mathrm{~nm}) / \mathrm{DBP}: \mathrm{C}_{70}(x \mathrm{~nm}, 1: 10$ ratio by volume)/bathophenanthroline (BPhen) $(8 \mathrm{~nm}) / \mathrm{Ag}$ cathode $(100 \mathrm{~nm})$. The 1:10 DBP: $\mathrm{C}_{70}$ films were grown by VTE (base pressure $\sim 2 \times 10^{-7}$ torr) at deposition rates of $0.2 \AA / \mathrm{s}$ and $2.0 \AA / \mathrm{s}$, and by OVPD using source temperatures of $375 \pm 2{ }^{\circ} \mathrm{C}$ and $540 \pm 1{ }^{\circ} \mathrm{C}$ for $\mathrm{DBP}$ and $\mathrm{C}_{70}$, respectively. For OVPD, a $\mathrm{N}_{2}$ carrier at a mass flow rate of 50 standard cubic centimeters per minute $(\mathrm{sccm})$ was simultaneously injected into both source cells using the system configuration described previously. ${ }^{[8,17,18]}$ Device performance as a function of mixed active layer thickness, $x$, in Figure 1, indicates that for $x>60 \mathrm{~nm}$, the FF of VTE-grown devices dramatically decreases. Consequently, at $x=60 \mathrm{~nm}$ the VTE-grown device has a maximum $P C E=6.2 \pm 0.2 \%$ (at 1 sun, AM1.5G illumination, spectrally corrected) and $F F=0.58 \pm 0.01$. On the other hand, OVPD-grown devices have $F F=0.61 \pm 0.01$ up to $x=100 \mathrm{~nm}$, and the short-circuit current density, $J_{\mathrm{sc}}$, peaks at $x=100 \mathrm{~nm}$, leading to $P C E=6.7 \pm 0.2 \%$ and $F F=0.61 \pm 0.01$. These trends are also apparent in the $J-V$ characteristics under similar illumination conditions shown in Figure 2a, where devices grown by VTE and OVPD are compared at the optimum thicknesses of $x=60 \mathrm{~nm}$ and $100 \mathrm{~nm}$, respectively. Figure $2 \mathrm{~b}$ shows the external and internal quantum efficiency (EQE, IQE, respectively) spectra of these devices. Here, IQE is obtained from the EQE and the absorption spectra. ${ }^{[24,25]}$ The $x=100 \mathrm{~nm}$ OVPD-grown device has a $\sim 7 \%$ higher IQE than the $x=60 \mathrm{~nm}$ device grown by VTE. Since IQE is the ratio of charge at the contacts to the number of photons absorbed within the active layer, ${ }^{[25]}$ OVPD-grown OPVs exhibit higher efficiency charge transfer than analogous VTE-grown devices.

The DBP: $\mathrm{C}_{70}$ mixed layers were examined using atomic force microscopy (AFM) and transmission electron microscopy (TEM) to determine the morphologies achieved by the two growth techniques. Figure 3a, shows an AFM image of a VTEgrown film on a Si substrate. The surface is featureless with a root-mean-square roughness of $r m s=0.4 \pm 0.1 \mathrm{~nm}$, showing no indication of a crystalline nanostructure. In contrast, the OVPD-grown film (Figure $3 \mathrm{~b}$ ) exhibits $\sim 40 \mathrm{~nm}$ diameter features suggestive of nanocrystallites, with $r m s=1.5 \pm 0.2 \mathrm{~nm}$. The structures were also investigated by selected area electron 


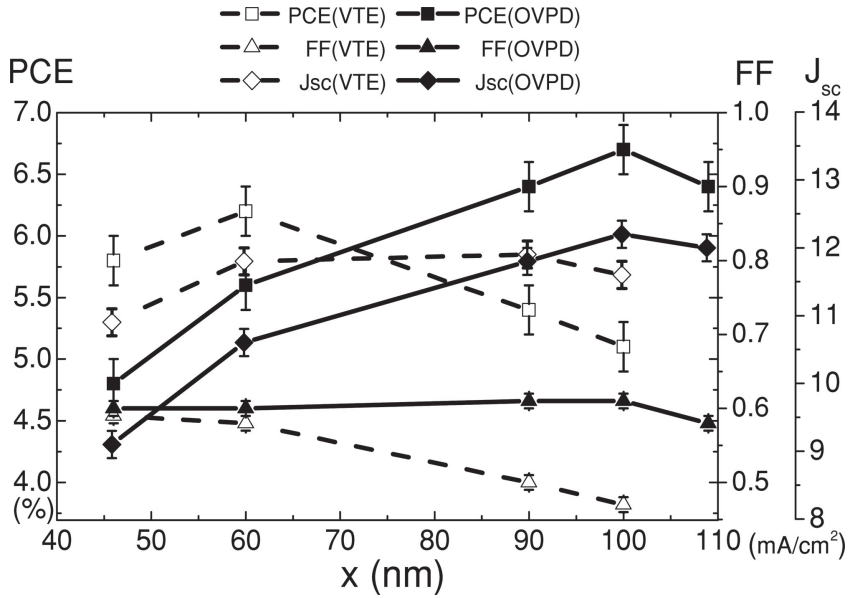

Figure 1. Power conversion efficiency $(P C E)$, fill factor $(F F)$, and shortcircuit current density $\left(U_{s c}\right)$ of tetraphenyldibenzoperiflanthen (DBP): $C_{70}$ mixed heterojunction devices grown by vacuum thermal evaporation (VTE) and organic vapor phase deposition (OVPD) as functions of mixed layer thickness. Open circuit-voltages for all devices are $0.90 \pm 0.01 \mathrm{~V}$. Standard deviations of device parameters $\left(U_{s c}, F F, P C E\right)$ in are $\pm 0.2 \mathrm{~mA} / \mathrm{cm}^{2}$, $\pm 0.01, \pm 0.2 \%$, respectively.

diffraction (SAED), as shown in Figures 3c and 3d, for VTEand OVPD-grown films, respectively. It has been reported that both hexagonal close packed (hcp) and face-centered cubic (fcc) polymorphs of $\mathrm{C}_{70}$ can co-exist, with their ratio dependent on the growth temperature and pressure. ${ }^{[26-28]}$ The fcc polymorph of $\mathrm{C}_{70}$ has a unit cell dimension of $a=14.89 \AA .^{[29]}$ The SAED pattern of the VTE-grown film (Figure 3c) indicates a completely amorphous layer, which is in striking contrast to the OVPD-grown film in Figure $3 \mathrm{~d}$, where four concentric rings corresponding to diffraction peaks ${ }^{[2,30]}$ suggestive of fcc $C_{70}$. Identification of the peaks in the figure and their interplanar spacings are summarized in Table 1 . We attribute the crystallinity of $\mathrm{C}_{70}$ observed in OVPD-grown films to the presence of $\mathrm{N}_{2}$ molecules that impart energy during growth. The carrier gas enhances surface diffusion of the incident organic molecules that promotes nucleation, desorption, crystallite ripening, etc., resulting in a morphology that approaches that of the equilibrium crystal structure. ${ }^{[10,18]}$
While SAED does not provide information on the arrangement of the DBP molecules in such dilute mixtures as employed here, the differences in nanostructure result in changes in spectral properties from which the structure may be indirectly inferred. As shown in Figure $4 \mathrm{a}$, the OVPD-grown DBP: $\mathrm{C}_{70}$ film has a lower extinction coefficient $(k)$ in the DBP spectral range between wavelengths of $\lambda=500 \mathrm{~nm}$ and $625 \mathrm{~nm}$, ${ }^{[15,31,32]}$ compared to that grown by VTE. Anisotropic materials typically have at least two different optical constants: Ordinary optical constants for p-polarized light and extraordinary optical constants for s-polarizations. ${ }^{[33]}$ The ordinary extinction coefficient for the planar DBP molecule is larger than the extraordinary coefficient, and hence the absorption of DBP depends on its preferred orientation in the film, ${ }^{[31]}$ which in turn is strongly affected by the substrate and growth process. ${ }^{[3,34]}$ In our case, we attribute the decreased extinction coefficient in OVPDgrown films to a preferred molecular orientation along the axis of lower $k$ relative to the light that is incident normal to the substrate. As a result, OVPD-grown OPVs require a thicker mixed layer than an analogous VTE-grown layer to achieve a similar EQE, as shown in Figure 1. Hence, we conclude that the nanocrystalline morphology of $\mathrm{C}_{70}$ affects the orientation of the diluted DBP when grown by OVPD, whereas this preferred orientation is not observed in VTE growth.

Since the nanostructure of the organic film affects the allowed optical transitions near the highest occupied molecular orbital (HOMO) - lowest unoccupied molecular orbital (LUMO) energy gap (i.e. near the long wavelength optical cutoff), ${ }^{[35,36]}$ we can analyze the spectral properties of the films in this region to gain further information about their morphologies. That is, structural disorder leads to a broadened density of states that results in an Urbach tail near the absorption cutoff ${ }^{[37-39]}$ that follows: $\alpha=\alpha_{0} \exp (h c /(\lambda U))$. Here, $\alpha_{0}$ is a constant, $h$ is Planck's constant, $c$ is the speed of light, and $U$ is the Urbach tail energy (see Figure $4 \mathrm{~b}$ ). From the slopes of the best fits to the data, we obtain $U=81 \pm 2 \mathrm{meV}$ and $101 \pm 4 \mathrm{meV}$ for $\mathrm{DBP}: \mathrm{C}_{70}$ films grown by OVPD and VTE, respectively, compared to the reported value of VTE-grown $\mathrm{C}_{70}$ of $55 \mathrm{meV} .^{[38]}$ Errors in $U$ correspond to the standard deviation of four samples. The lower energy for OVPD indicates reduced disorder than for VTEgrown films. This also agrees with the observation that OVPD results in an ordered $\mathrm{C}_{70}$ fcc structure (c.f. Figure 3).
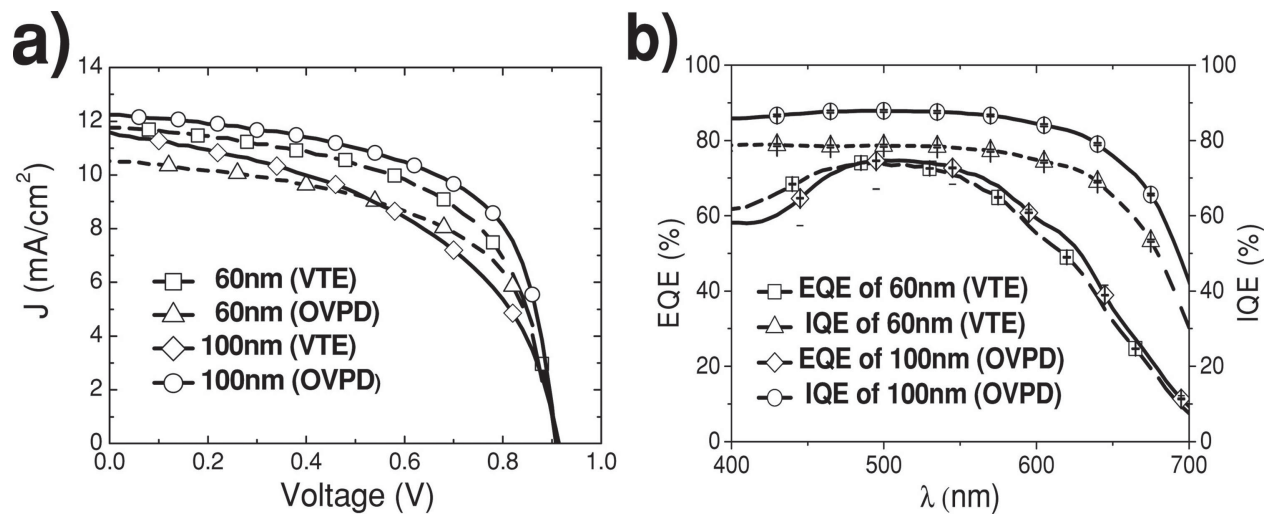

Figure 2. (a) Current density $(J)$ versus voltage $(V$ characteristics under 1 sun illumination for $60 \mathrm{~nm}$ or $100 \mathrm{~nm}$ active layer thicknesses grown by VTE and OVPD, respectively. (b) External (triangles) and internal (squares) quantum efficiencies as function of wavelength $(\lambda)$ of the devices in (a). 


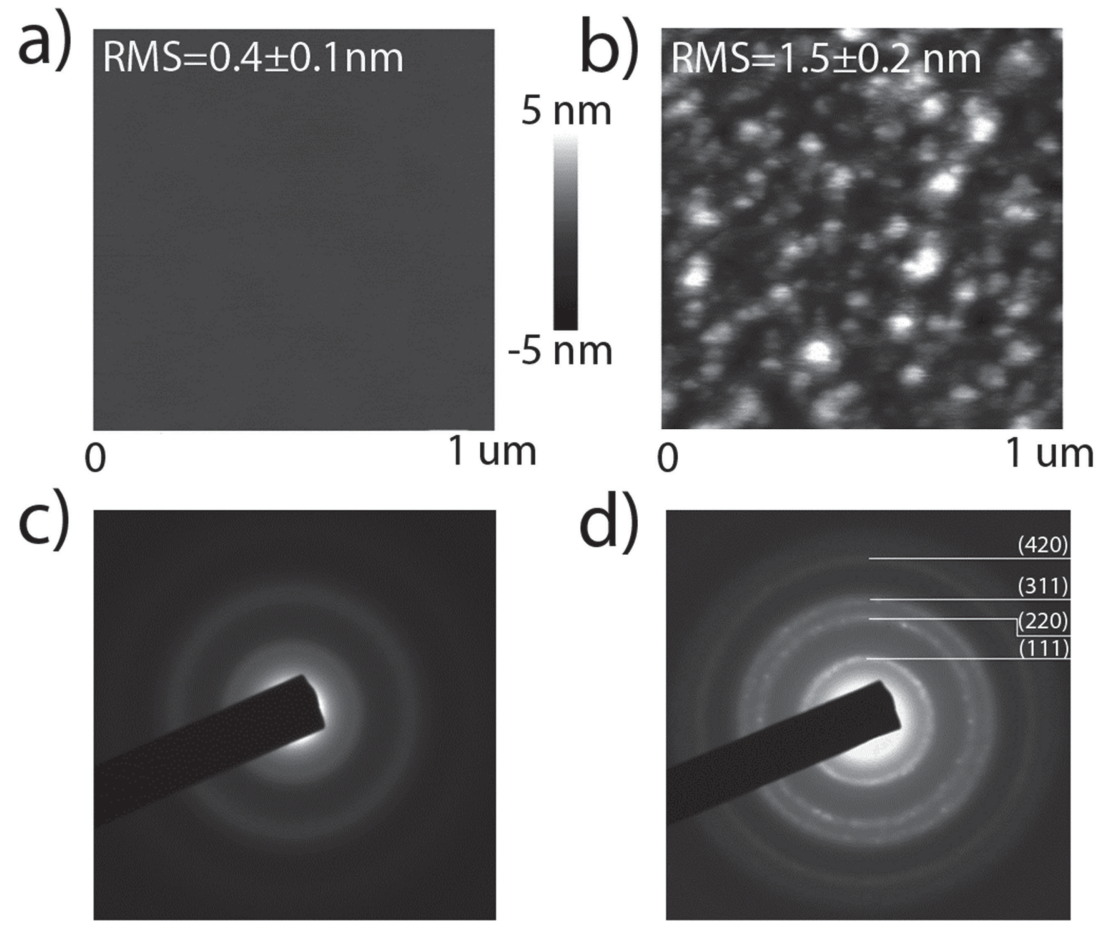

Figure 3. Atomic force microscopy (AFM) images of $60 \mathrm{~nm}$ thick $D B P: C_{70}$ (1:10 ratio) films grown by (a) VTE and (b) OVPD on a Si substrate. The root-mean-square (RMS) roughness of the films is shown. The errors are due to variation from area to area of the film. Selected area electron diffraction (SAED) patterns of DBP: $C_{70}$ films grown by (c) VTE and (d) OVPD. Corresponding Miller indices of $\mathrm{C}_{70}$ face-centered cubic (fcc) structure are indicated for the OVPD-grown film. Both images were taken for a $20 \mu \mathrm{m}$ diameter selected area aperture at beam current density of $20 \mathrm{pA} / \mathrm{cm}^{2}$.

Finally, the $J-V$ characteristics of the OPVs can be understood in terms of the modified ideal-diode equation for organic heterojunctions: ${ }^{[40,41]}$

$$
\begin{aligned}
J= & J_{\mathrm{sD}}\left[\exp \left(\frac{q\left(V-J R_{s}\right)}{n_{D} k_{b} T}\right)-\chi\right] \\
& +J_{\mathrm{sA}}\left[\exp \left(\frac{q\left(V-J R_{s}\right)}{n_{A} k_{b} T}\right)-\chi\right]-q \eta_{P P d} J_{X}
\end{aligned}
$$

Here, $J_{\mathrm{sD}}$ and $J_{\mathrm{sA}}$ are the saturation current densities associated with trapped charge in the donor and acceptor layers, respectively, $n_{\mathrm{D}}$ and $n_{\mathrm{A}}$ are the corresponding ideality factors,

Table 1. Interplanar d-spacings of OVPD grown DBP: $C_{70}$ films.

\begin{tabular}{lccc}
\hline (hkl) index & $\mathrm{d}=\mathrm{a} / \mathrm{a} \sqrt{\mathrm{h}^{2}+\mathrm{k}^{2}+\mathrm{l}^{2}}$ & $d_{1} / d^{\mathrm{b})}($ XRD) & $d_{1} / d$ (SAED) \\
\hline$(111)$ & $8.59 \AA$ & 1.00 & 1.00 \\
$(220)$ & $5.26 \AA$ & 1.63 & 1.62 \\
$(311)$ & $4.49 \AA$ & 1.91 & 1.93 \\
$(420)$ & $3.39 \AA$ & 2.53 & 2.60 \\
\hline
\end{tabular}

a) $\mathrm{a}=14.89 \AA$ is the lattice constant of the $C_{70}$ fcc structure in. $d_{1}$ corresponds to the (111) plane. The errors in $d_{1} / d$ from SAED $\sim 3 \%$ arise from inaccuracies in extracting diameters of concentric rings in Figure $3 \mathrm{~d}$; b) $\mathrm{C}_{70}$ data from Ref. [30]
$J_{X}$ is the exciton current density, $R_{s}$ is the series resistance, $q$ is electron charge, $T$ is the temperature, $k_{b}$ is Boltzmann's constant, and $\eta_{P P d}$ is the polaron pair dissociation efficiency. Also, $\chi$ is the ratio of the polaron pair dissociation rate at voltage, $V$, to its value at equilibrium. For simplicity, we assume $\chi \approx 1 .{ }^{[41]}$ The fit to the forward characteristics of the devices in Figure 1 measured at their optimal thicknesses are shown in Figure 5a, from which we obtain $R_{\mathrm{s}}$ vs. $x$ plotted in Figure $5 \mathrm{~b}$, along with the corresponding FF. While $R_{s}$ for VTE -grown devices increases linearly from $0.5 \pm 0.1$ at $x=45 \mathrm{~nm}$ to $2.5 \pm 0.2 \Omega-\mathrm{cm}^{2}$ at $x=100 \mathrm{~nm}$, its value for OVPD-grown devices is nearly thickness independent, with $R_{s}<0.5 \pm 0.1 \Omega$ - $\mathrm{cm}^{2}$ up to $x=110 \mathrm{~nm}$. The decreased series resistances is primarily due to the improved crystallinity of $\mathrm{C}_{70}$ (see Figure 3 ). Furthermore, a directional grain structure of DBP molecules has previously been observed for substrates heated during growth. ${ }^{[42]}$ Since OVPD employs a hot carrier gas, the temperature of the growth surface can be higher than in VTE growth. Further, oriented films of DBP may have a higher surface mobility of molecules, also enabled by the presence of the carrier gas. This directionality in film growth leads to an equilibrium nanocrystalline structure that increases charge mobility. ${ }^{[43]}$ Both the improved crystallinity of $\mathrm{C}_{70}$ and preferential orientation of DBP explain that OVPD growth results in a morphology that leads to improved charge extraction compared to the amorphous VTE-grown structures.

In conclusion, the growth of mixed $\mathrm{DBP}: \mathrm{C}_{70}$ layers by OVPD leads to a nanocrystalline morphology that improves charge extraction and reduced charge recombination in mixed heterojunction organic photovoltaic cells relative to analogous cells grown by VTE. Since OVPD growth occurs near thermodynamic equilibrium, mixed layers are nanocrystalline compared with amorphous layers grown by the highly nonequilibrium process of VTE. A consequence of the enhanced crystallinity is a reduction in the extinction coefficient of DBP. The enhanced crystallinity also results in a low series resistance and, hence, a high FF even for relatively thick mixed $\mathrm{DBP}: \mathrm{C}_{70}$ films. The absorption loss in the nanocrystalline films is compensated by the use of thicker active layers without negatively impacting either $R_{\mathrm{S}}$ or FF. Optimal $\mathrm{DBP}: \mathrm{C}_{70}$ layers grown by OVPD have a thickness of $100 \mathrm{~nm}$, resulting in OPVs with $P C E=6.7 \pm 0.2 \%$. This compares to $P C E=6.2 \pm 0.2 \%$ obtained with optimized $60 \mathrm{~nm}$-thick mixed layers grown by VTE. The significantly thicker nanocrystalline devices should ultimately result in higher device yields through the reduction 

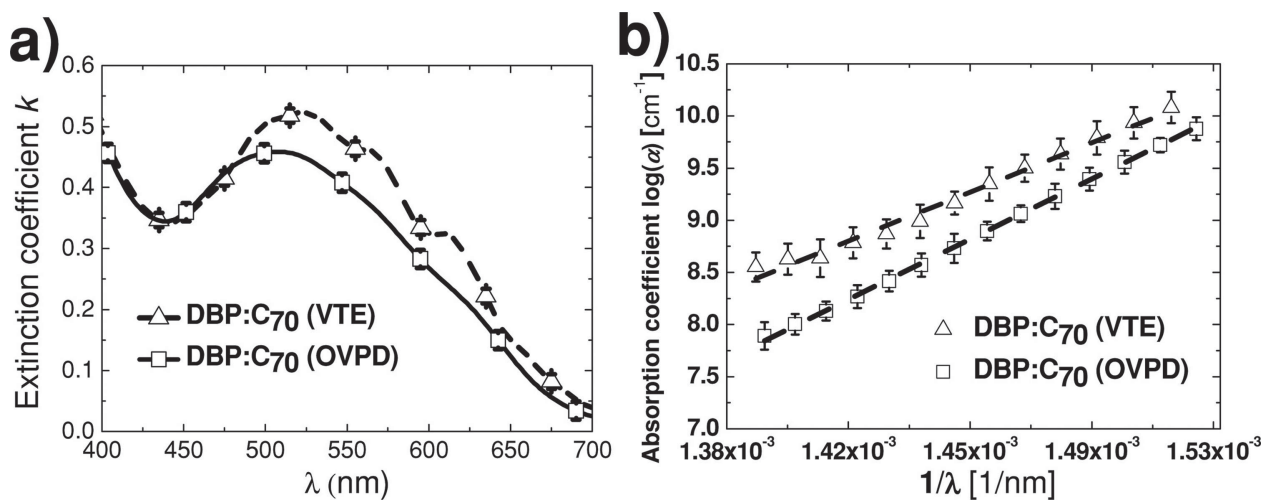

Figure 4. (a) Extinction coefficient ( $k$ ) of DBP: $C_{70}$ grown by VTE and OVPD as a function of wavelength ( $\lambda$ ). (b) Natural logarithm of the absorption coefficient $(\log (\alpha))$ of DBP: $C_{70}$ films grown by VTE and OVPD, vs. $1 / \lambda$ near the film long wavelength cutoff. Dashed lines are linear fits to the data. Their slopes yield the Urbach tail energies, $U=101 \pm 4 \mathrm{meV}$ and $81 \pm 2 \mathrm{meV}$ for VTE and OVPD-grown mixtures, respectively.

of pin-holes and other shunt paths often observed in very thin VTE-grown OPVs.

\section{Experimental Section}

Device Fabrication: Glass substrates pre-coated with a $70 \mathrm{~nm}$ thick layer of indium tin oxide (ITO) having a sheet resistance of $20 \Omega / \square$ were cleaned with tergitol, deionized water, acetone, and isopropanol, followed by exposure to ultraviolet-ozone treatment for $10 \mathrm{~min}$. The substrates were transferred into a vacuum thermal evaporation chamber to deposit $\mathrm{MoO}_{3}$ at $1.0 \AA \mathrm{A}$ s. The $\mathrm{DBP}: \mathrm{C}_{70}$ mixed layers were grown on $\mathrm{MoO}_{3}$ by either VTE or OVPD, transferring the samples into the respective growth chamber through an ultrahigh purity $\mathrm{N}_{2}$-filled glove box. The remaining layers of BPhen and Ag were deposited by VTE, also following transfer from the OVPD reactor without air exposure. For VTE growth, two separate quartz crystal monitors tracked the deposition rates of $D B P$ and $C_{70}$, at $0.2 \AA / s, 2.0 \AA / s$ (1:10 ratio), respectively at base pressure of $\sim 10^{-7}$ torr. For OVPD, the three zones of the reactor were set a 570,510 , and $450^{\circ} \mathrm{C}$, respectively, creating a temperature gradient from the source to the substrate positions along the tube. The DBP and $C_{70}$ were co-evaporated by heating the source materials to $375 \pm 2{ }^{\circ} \mathrm{C}$ and, $540 \pm 1{ }^{\circ} \mathrm{C}$ respectively, at a source barrel flow rate of $50 \mathrm{sccm} \mathrm{N}$. The gas solvent was further diluted by $20 \mathrm{sccm} \mathrm{N} N_{2}$ introduced directly into the main reactor tube. The pressure during growth was maintained at 0.61 torr. These conditions achieved the same deposition rate as in VTE.

Measurement of Device Characteristics: $J-V$ characteristics were obtained in an ultrahigh purity $\mathrm{N}_{2}$ gas environment using an Agilent $4156 \mathrm{C}$ semiconductor parameter analyzer. Simulated AM 1.5C illumination, 1 sun intensity $\left(100 \mathrm{~mW} / \mathrm{cm}^{2}\right)$ was provided using a solar simulator, and calibrated using a standard Si reference cell traceable to National Renewable Energy Laboratory (NREL) standards. The EQE spectra were measured with a Stanford SR830 DSP lock-in amplifier under a $200 \mathrm{~Hz}$-chopped monochromated Xe-lamp. A NIST-traceable Si detector was used as a reference.

Selective Area Electron Diffraction (SAED) measurements: Organic layers were deposited onto $\mathrm{MoO}_{3}(10 \mathrm{~nm})$ pre-deposited on $\mathrm{S}$ substrates cleaned with acetone and isopropanol. Organic layers were captured on a copper grid by dissolving $\mathrm{MoO}_{3}$ in deionized water The SAED patterns were recorded using a JEOL 3011 high resolution electron microscope at an accelerating voltage of $300 \mathrm{kV}$ with a $20 \mu \mathrm{m}$ selective aperture diameter.

Measurement of Optical Constants: Variable angle spectroscopic ellipsometery was used to measure thickness and optical constants of thin film samples deposited on Si substrates. Spectroscopic data were recorded in the near infrared for thickness measurements, and in the ultraviolet-visible range for obtaining the optical constants, using a B-spline algortithm. ${ }^{[44]}$ This procedure was repeated over samples having different thicknesses to reduce error.
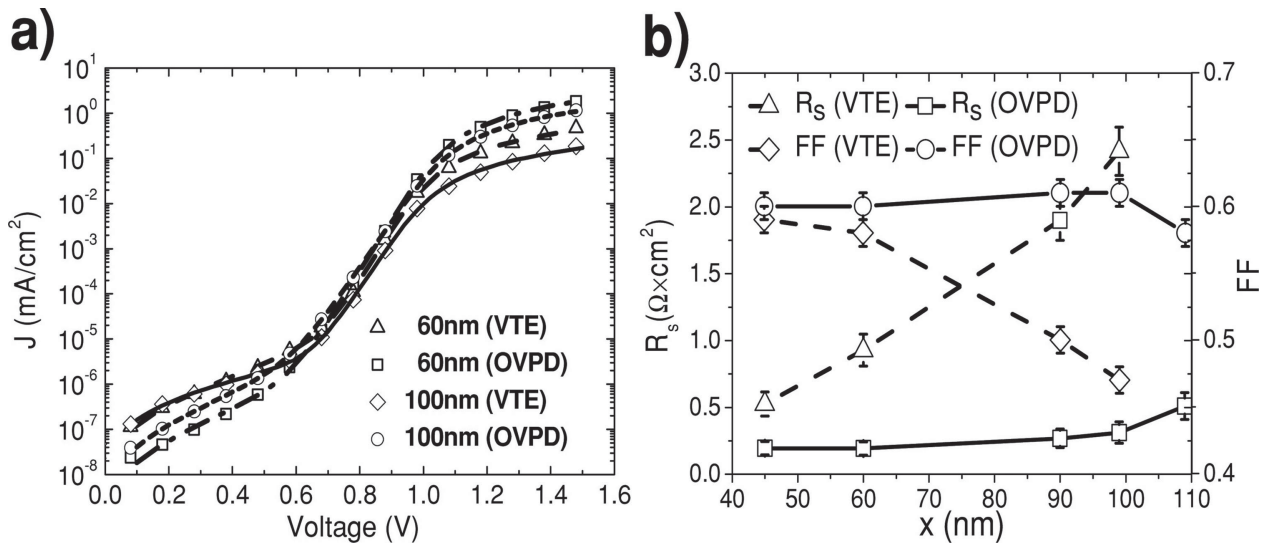

Figure 5. (a) Fits of the forward-biased $J-V$ characteristics of the devices in Figure 1 using the ideal diode equation for excitonic junctions for $60 \mathrm{~nm}$ and $100 \mathrm{~nm}$ thick active layer OPVs grown by VTE and OVPD, respectively. (b) Series resistance $\left(R_{\mathrm{S}}\right)$ and fill factor (FF) for devices in (a). Error bars correspond to the standard deviation of 4 devices for each data point. 


\section{Acknowledgements}

The authors gratefully acknowledge Mr. Anurag Panda, Mr. Xin Xiao, Mr. Kyusang Lee, Dr. Olga Griffith, and Mr. Sungyong Seo for useful suggestions and discussions. This work was partially supported by the SunShot Program of the US Department of Energy (EERE) under award number DE-EE0005310 (BS, experiment, analysis, CR, OVPD growth). We are also grateful to the U.S. Department of Energy (DOE), Office of Basic Energy Science, as part of the Center for Solar and Thermal Energy Conversion, Energy Frontier Research Center, grant award DE-SC0000957 (JDZ, diffraction analysis) and NanoFlex Power Inc (SRF, experimental direction, analysis).

Received: October 13, 2013

Revised: December 7, 2013

Published online: February 8, 2014

[1] S. R. Forrest, Nature 2004, 428, 911.

[2] W. Zeng, K. S. Yong, Z. M. Kam, F. Zhu, Y. Li, Appl. Phys. Lett. 2010, 97, 133304.

[3] G. D. Wei, S. Y. Wang, K. Sun, M. E. Thompson, S. R. Forrest, Adv. Energy Mater. 2011, 1, 184.

[4] O. P. Lee, A. T. Yiu, P. M. Beaujuge, C. H. Woo, T. W. Holcombe, J. E. Milestone, J. D. Douglas, M. S. Chen, J. M. J. Frechet, Adv. Mater. 2011, 23, 5359.

[5] J. D. Zimmerman, X. Xiao, C. K. Renshaw, S. Y. Wang, V. V. Diev, M. E. Thompson, S. R. Forrest, Nano Lett. 2012, 12, 4366.

[6] M. Baldo, M. Deutsch, P. Burrows, H. Gossenberger, M. Gerstenberg, V. Ban, S. Forrest, Adv. Mater. 1998, 10, 1505.

[7] M. Shtein, P. Peumans, J. B. Benziger, S. R. Forrest, J. Appl. Phys. 2003, 93, 4005

[8] F. Yang, K. Sun, S. R. Forrest, Adv. Mater. 2007, 19, 4166.

[9] A. Farahzadi, P. Niyamakom, M. Beigmohamadi, N. Meyer, D. Keiper, M. Heuken, F. Ghasemi, M. R. Rahimi Tabar, T. Michely, M. Wuttig, EPL (Europhysics Letters) 2010, 90, 10008.

[10] C. Rolin, K. Vasseur, B. Niesen, M. Willegems, R. Müller, S. Steudel, J. Genoe, P. Heremans, Adv. Funct. Mater. 2012, 22, 5050

[11] C. Rolin, G. Vartanian, S. R. Forrest, J. Appl. Phys. 2012, 112, 113502.

[12] R. R. Lunt, B. E. Lassiter, J. B. Benziger, S. R. Forrest, Appl. Phys. Lett. 2009, 95, 233305

[13] C. Rolin, K. Vasseur, J. Genoe, P. Heremans, Org. Electron. 2010, 11, 100.

[14] J. Xue, B. P. Rand, S. Uchida, S. R. Forrest, Adv. Mater. 2005, $17,65$.

[15] X. Xiao, J. D. Zimmerman, B. E. Lassiter, K. J. Bergemann, S. R. Forrest, Appl. Phys. Lett. 2013, 102, 073302.

[16] J. Xue, B. P. Rand, S. Uchida, S. R. Forrest, J. Appl. Phys. 2005, 104, 124903.

[17] F. Yang, M. Shtein, S. R. Forrest, Nat. Mater. 2004, 4, 37.

[18] F. Yang, M. Shtein, S. R. Forrest, J. Appl. Phys. 2005, 98, 014906.

[19] C. H. Peters, I. T. Sachs-Quintana, J. P. Kastrop, S. Beaupré, M. Leclerc, M. D. McGehee, Adv. Energy Mater. 2011, 1, 491.
[20] F. C. Krebs, Stability and Degradation of Organic and Polymer Solar Cells, Wiley, New York, 2012

[21] K. Feron, T. J. Nagle, L. J. Rozanski, B. B. Gong, C. J. Fell, Solar Energy Materials and Solar Cells 2013, 109, 169.

[22] M. Schwambera, N. Meyer, M. Gersdorff, M. Reinhold, G. Strauch, R. Beccard, M. Heuken, SID Symposium Digest of Technical Papers 2003, 34, 1419.

[23] B. Marheineke, Proc. of SPIE 2005, 5961, 596101.

[24] L. A. A. Pettersson, L. S. Roman, O. Inganäs, J. Appl. Phys. 1999, 86, 487.

[25] P. Peumans, A. Yakimov, S. R. Forrest, J. Appl. Phys. 2003, 104, 3693.

[26] G. B. Vaughan, P. A. Heiey, D. E. Luzzi, D. A. Ricketts-Foot, A. R. McGhie, J. E. Fischer, Y. W. Hui, A. L. Smith, D. E. Cox, W. J. Romanow, B. H. Allen, N. Coustel, J. P. McCauley, A. B. Smith, Science (New York, N.Y.) 1991, 254, 1350.

[27] G. Vantendeloo, S. Amelinckx, S. Muto, M. Verheijen, P. Vanloosdrecht, G. Meijer, Ultramicroscopy 1993, 51, 168.

[28] V. D. Blank, G. A. Dubitsky, N. R. Serebryanaya, B. N. Mavrin, V. N. Denisov, S. G. Buga, L. A. Chernozatonskii, Physica B: Condensed Matter 2003, 339, 39.

[29] M. Valsakumar, N. Subramanian, M. Yousuf, P. Sahu, Y. Hariharan, A. Bharathi, V. S. Sastry, J. Janaki, G. Rao, T. Radhakrishnan, C. Sundar, Phys. Rev. B 1993, 48, 9080

[30] M. C. Valsakumar, N. Subramanian, M. Yousuf, P. C. Sahu, Y. Hariharan, A. Bharathi, V. S. Sastry, J. Janaki, G. V. N. Rao, T. S. Radhakrishnan, C. S. Sundar, Pramana-journal of Physics 1993, 40, L137.

[31] D. Yokoyama, Z. Q. Wang, Y. Pu, K. Kobayashi, J. Kido, Z. Hong, Solar Energy Materials and Solar Cells 2012, 98, 472.

[32] Z. Wang, D. Yokoyama, X. Wang, Z. Hong, Y. Yang, J. Kido, Energy \& Environmental Science 2013, 6, 249.

[33] D. Yokoyama, J. Mater. Chem. 2011, 21, 19187.

[34] S. R. Forrest, Chem. Rev. 1997, 97, 1793.

[35] M. S. Dresselhaus, G. Dresslhaus, Ann. Rev. Mater. Sci. 1995, 25, 487.

[36] H. Kataura, Y. Endo, K. Kikuchi, T. Hanyu, S. Yamaguchi, J. Phys. and Chem. Sol. 1997, 58, 1913.

[37] T. Gotoh, S. Nonomura, S. Hirata, S. Nitta, Appl. Surf. Sci. 1997, 113/114, 278.

[38] W. Zhou, S. Xie, S. Qian, T. Zhou, R. Zhao, G. Wang, L. Qian, W. Li, J. Appl. Phys. 1996, 104, 459.

[39] H. Habuchi, S. Nitta, D. X. Han, S. Nonomura, J. Appl. Phys. 2000, 87,8580 .

[40] B. P. Rand, D. P. Burk, S. R. Forrest, Phys. Rev. B 2007, 75, 115327.

[41] N. C. Giebink, G. P. Wiederrecht, M. R. Wasielewski, S. R. Forrest, Phys. Rev. B 2010, 82, 155305.

[42] Y. Zhou, T. Taima, Y. Shibata, T. Miyadera, T. Yamanari, Y. Yoshida, Solar Energy Materials and Solar Cells 2011, 95, 2861.

[43] D. Yokoyama, Y. Setoguchi, A. Sakaguchi, M. Suzuki, C. Adachi, Adv. Funct. Mater. 2010, 20, 386

[44] J. W. Webber, T. A. R. Hansen, M. C. M. van de Sanden, R. Engeln, J. Appl. Phys. 2009, 106, 123503. 